\title{
Epigenetik - neue Erkenntnisse zum Verständnis neurodegenerativer Erkrankungen
}

\author{
Epigenetic Mechanisms in Neurodegenerative Diseases
}

Autoren

Institute
A. Fischer ${ }^{1,2}$, A. Schneider ${ }^{1,2}$, U. Wüllner ${ }^{1,3}$

${ }^{1}$ Deutsches Zentrum für neurodegenerative Erkrankungen (DZNE)

2 Psychiatrie und Psychotherapie, Universitätsmedizin Göttingen

${ }^{3}$ Neurologie, Universität Bonn
Schlüsselwörter

- Epigenetik

DNA-Methylierung

- microRNA

- Parkinson

- Alzheimer

Keywords

- epigenetics

- DNS-methylation

- micro RNA

- Parkinson
- Alzheimer

\section{Zusammenfassung \\ $\nabla$}

Epigenetik bezeichnet die verschiedenen, über die DNA-Sequenz hinausgehenden Strukturen und Funktionen der DNA-Protein-Matrix (Chromatin), die die Genexpression regulieren. Dieses Epigenom bestimmt die unterschiedliche Entwicklung, die genetisch identische Zellen und Individuen (eineiige Zwillinge) nehmen. Epigenetische Merkmale wie z.B. die direkte biochemische Modifikationen der DNA durch Methylierung können durch Umwelteinflüsse verändert und in veränderter Form vererbt werden, ohne dass die Sequenz der DNA verändert wird. Die Analyse epigenetischer Prozesse, insbesondere der DNAMethylierung, der Modifikationen der DNA-bindenden Histon-Proteine und der Funktionen der nicht kodierenden microRNA, eröffnet ein unerwartet komplexes Feld von Umwelt-Gen-Interaktionen, die die individuellen Ausprägungen von Krankheitsmerkmalen ebenso beeinflussen wie Wirkungen und Nebenwirkungen vieler Medikamente.

Die Erforschung des Epigenoms wird auch für die beiden häufigsten neurodegenerativen Erkrankungen, die Alzheimer'sche und Parkinson'sche Krankheit, neue diagnostische und möglicherweise auch therapeutische Perspektiven aufzeigen.

\section{Einleitung}

Dol http://dx.doi.org/

10.1055/s-0035-1552630

Akt Neurol 2015; 42: 393-401

(c) Georg Thieme Verlag KG

Stuttgart · New York

ISSN 0302-4350

Korrespondenzadresse

Prof. Ullrich Wüllner

Neurologie, Universität Bonn

Sigmund-Freud-Str. 25

53105 Bonn

wuellner@uni-bonn.de

\section{$\nabla$}

Ein wesentliches Ergebnis des Humangenomprojekts war die Erkenntnis, dass angesichts der verhältnismäßig kleinen Zahl von identifizierten Genen noch andere Mechanismen, über die eigentliche Sequenzinformation hinaus, zu der großen Variabilität der Genexpression in menschlichen Zellen beitragen müssen. So kann z.B. die DNASequenzinformation alleine keine Begründung für die unterschiedlichen Entwicklungen liefern,

\section{Abstract \\ $\nabla$}

Epigenetics describes the various structures and functions of the DNA-protein matrix (chromatin), which regulate gene expression beyond the DNA sequence. The epigenome determines the differences in the development of genetically identical cells and individuals (mono-zygotic twins). Epigenetic features such as direct biochemical modifications of DNA (methylation) are affected by environmental conditions (famine, early life stress) and are inherited in a modified form, without alteration of the DNA sequence. The analysis of epigenetic processes, in particular DNA methylation, modifications of the DNA-binding proteins, and the functions of the non-coding microRNA, opens an unexpectedly complex field of environment-gene interactions that affect the individual patterns of disease symptoms as well as effects and side effects of many drugs.

The study of the epigenome hold the promise of new diagnostic and possibly therapeutic perspectives also for the two most common neurodegenerative diseases, namely Alzheimer's and Parkinson's disease. 


\section{DNA-Methylierung}

1. DNA-Methylierung begünstigt die Deazetylierung von Histonen und induziert somit die Kondensation des Chromatins.

2. durch DNA-Methylierung werden 5-Methylcytosin-Bindeproteine rekrutiert, die als Repressoren dienen (z.B. Methyl-CpG-binding protein, $\mathrm{MeCP}-2$, s. u.)

3. Viele Transkriptionsfaktoren können nicht an $\mathrm{mCpG}$ binden

4. intragenische $\mathrm{mCpG}$ hemmen die korrekte Bindung der DNA-Polymerasen und verhindern damit aberrante Transkription

Der Begriff der Epigenetik wurde in den 40er-Jahren von dem britischen Biologen Conrad Hal Waddington (1905-1975) geprägt, der damit Epigenese, eine Bezeichnung für die Entwicklung des Embryos, und Genetik zusammenfassen wollte, um das rätselhafte Verhältnis von genetischer Veranlagung und phänotypischer Ausprägung zu beschreiben. Epigenetik im aktuellen Sprachgebrauch bezeichnet die verschiedenen, über die DNA-Sequenz hinausgehenden Strukturen und Funktionen der DNAProtein-Matrix (Chromatin), die die Genexpression regulieren. Das Epigenom bestimmt die unterschiedliche Entwicklung, die genetisch identische Zellen und Individuen (eineiige Zwillinge) nehmen. Epigenetische Merkmale wie z.B. die direkte biochemische Modifikation der DNA durch Methylierung können durch Umwelteinflüsse verändert und in veränderter Form vererbt werden, ohne dass die Sequenz der DNA verändert wird. Die moderne Epigenetik bestätigt die Theorien Lamarcks und eröffnet ein unerwartet komplexes Feld von Umwelt-Gen-Interaktionen, die vermutlich die individuellen Ausprägungen von Krankheitsmerkmalen ebenso beeinflussen wie Wirkungen und Nebenwirkungen vieler Medikamente.

In diesem Artikel werden im Folgenden neben der DNA-Methylierung und den Modifikationen der DNA-bindenden Histon-Proteine auch die Funktionen der nicht kodierenden RNAs, insbesondere der miRNA, skizziert und anschließend aufgezeigt, welche diagnostischen und möglicherweise auch therapeutischen Perspektiven sich aus der Erforschung des Epigenoms für die beiden häufigsten neurodegenerativen Erkrankungen, die Alzheimer'sche und Parkinson'sche Krankheit ergeben.

\section{DNA-Methylierung}

Die Methylierung von Zytosin zu 5-Methylcytosin (welches auch als „fünfte Base“ bezeichnet wird), findet in Bakterien, Pflanzen, Invertebraten und Vertebraten statt. In Säugetieren ist die Voraussetzung für die Methylierung von Zytosin ein direkt darauffolgendes Guanin. Diese Basenkombination wird als CpG-Dinukleotid bezeichnet und tritt im menschlichen Genom seltener auf, als es aufgrund des GC-Gehalts der DNA zu erwarten wäre. Am Anfang von Genen, in der Regel in den Promotorregionen findet man jedoch oft CpG-reiche Regionen, sog. CpG-Inseln (CGI). Die CpGs in den CGI sind in der Regel unmethyliert und erlauben in der Regel Genexpression, während die einzelnen, im übrigen Genom verstreuten CpGs (70-80\% aller im menschlichen Genom vorkommenden $\mathrm{CpG}$-Dinukleotide) methyliert sind. Diese methylierten $\mathrm{CpG}(\mathrm{mCpG}$ ) wirken durch verschiedene Mechanismen (u.a. durch mCpG-bindende Proteine) hemmend auf die Expressionsregulation [1]. Methylierung tritt, wenn auch in geringerem Umfang, auch an anderen Basen auf; dieses Phänomen ist aber noch weitgehend unerforscht.
Das Methylierungsmuster der DNA wird bei der Zellteilung zwar grundsätzlich weitergegeben, unterliegt nach neuesten Erkenntnissen jedoch einer Reihe von Einflussfaktoren und ist wesentlich dynamischer reguliert, als noch bis vor Kurzem angenommen. Diese epigenetische Information des Erbguts wird nicht über Generationen konserviert, sondern unterliegt in hohem Maße auch Umwelteinflüssen und endogenen, noch weitgehend unerforschten Mechanismen [2]. So wurde in einer explorativen Studie an DNA aus isolierten Kernen humaner kortikaler Nervenzellen gezeigt, dass verschiedene Gruppen von Genen charakteristische Verläufe altersabhängiger Veränderungen des Methylierungsstatus aufweisen [3]. Während die Methylierung mancher Gene über die Lebenszeit kontinuierlich zunahm, waren andere nur in den ersten Lebensmonaten verstärkt methyliert und eine dritte Gruppe zeigte einen drastischen Anstieg der Methylierung in der 5. Dekade. Weitere Belege für Umwelt- und Verhaltenseinflüsse auf den Methylierungsstatus (und möglicherweise auch auf andere epigenetische Mechanismen) stammen z.B. aus Studien des murinen Maternalverhaltens und vergleichenden Untersuchungen von Probanden, deren Mütter während der Schwangerschaft hungern mussten, im Vergleich zu ihren unter günstigeren Bedingungen herangewachsenen Geschwistern $[4,5]$.

\section{Histone und Histon-modifizierende Enzyme}

Biochemische Modifikationen der DNA und der Histone sind eng miteinander verwoben und beeinflussen sich wechselseitig.

Histone bilden die Basis des Protein-DNA-Komplexes im Chromatin. Vier Histone (H2a, 2b, 3 und 4) bilden das Nukleosom, um das eine DNA-Sequenz von 146 Basenpaaren „gewickelt“ ist - eng gepackte Nukleosomen, durch sog. Linker-Histone (H1) verbunden, erlauben, den langen DNA-Strang auf kleinem Raum zu „verpacken“. Biochemische Modifikationen der Histone an ihren aminoterminalen Abschnitten, die aus der globulären Region herausragen, sind wichtige Signalsequenzen, deren Aminosäurenabfolge und -modifikation eine komplexe Ebene der Regulation darstellt ( $\bullet$ Abb.1).

So werden z. B. Lysine in den aminoterminalen Histonabschnitten von Histonazetyltransferasen und Deazetylasen (HAT/HDAC) modifiziert. Diese Lysine sind in ihrer natürlichen Form positiv geladen und binden fest an die negativ geladene DNA, kondensieren die Nukleosomen und formen eine geschlossene Chromatinstruktur, die von den Transkriptionskomplexen, Repressor- und Aktivatorkomplexen nicht aktiviert werden können. Durch die Azetylierung wird diese positive Ladung entfernt, sodass die Bindung zur DNA aufgelockert wird und ein Transkriptionsrepressor- oder Aktivatorkomplex leichter binden kann. Auch hier offenbart die Natur eine ungeahnte Komplexität. Nicht die Bindung einzelner Transkriptionsfaktoren an die Erkennungsmotive, wie sie im Modellsystem (typischer Luciferase-Assay oder Elektromobilitäts-Assay, EMSA) häufig dargestellt wird, sondern ein orchestriertes Muster von aktivierenden und inhibierenden Transkriptionskomplexen, reguliert die Aktivität eines Gens, bzw. modifiziert sie, je nach funktionellem Zustand und Erfordernis. Die Komplexität dieser Prozesse, die charakteristisch für den funktionellen Zustand einer Zelle sind, zeigt sich z. B. bei der Analyse durch Chromatin-Immunopräzipitation (CHIP). Die Analyse epigenetischer Prozesse im ZNS ist besonders schwierig, da die Bedeutung der unterschiedlichen Zelltypen (Neurone, Astro-, Oligo- und Mikroglia) bislang unzureichend berücksichtigt wurde. 


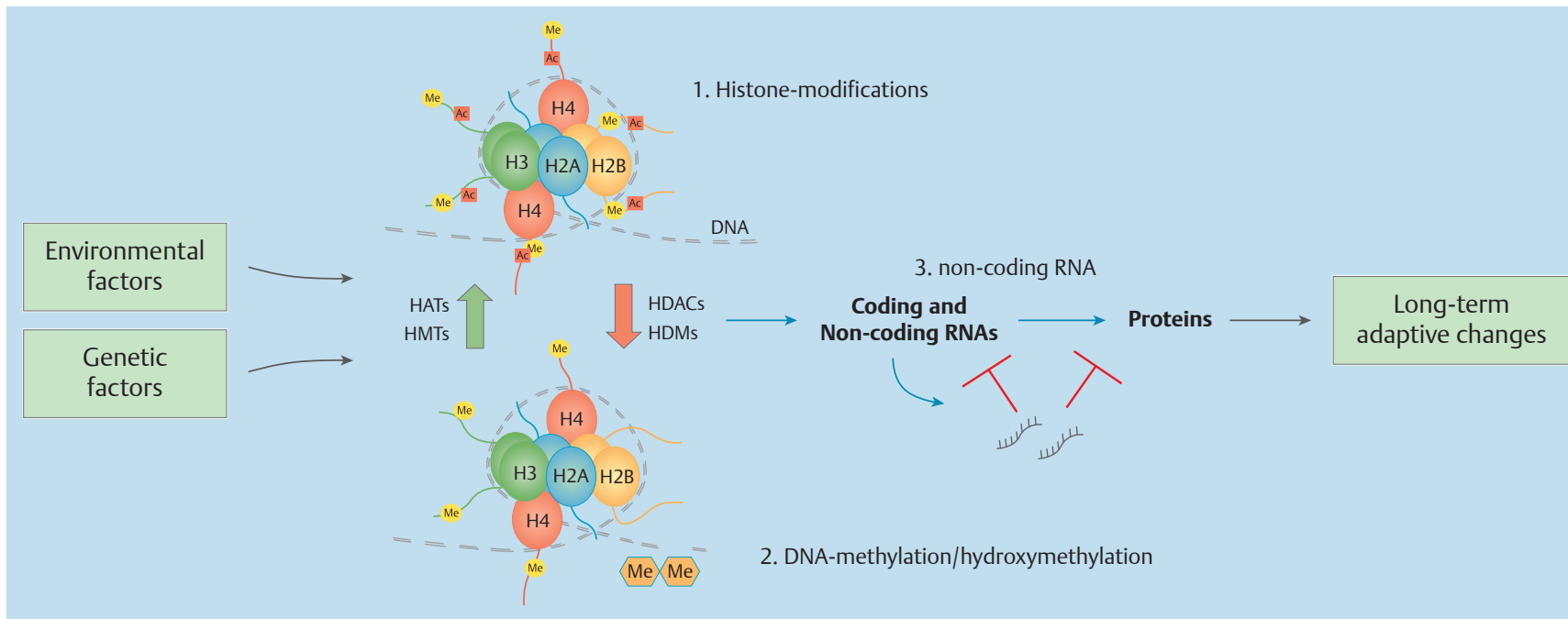

Abb.1 Histone können posttranslational verändert werden, z. B. durch das Anhängen von Azetylresten. Wenig azetylierte Histone bewirken dabei eine stark kondensierte DNA-Struktur, die in der Regel nicht für die Transkriptionsmaschinerie zugänglich und daher inaktiv ist. Die Bedeutungen der biochemischen Modifikationen der Histone sind nur teilweise bekannt.

Die posttranslationalen biochemischen Modifikationen der Histone sind bislang nur in Ansätzen untersucht. Obwohl die Einteilung und Identifizierung des Euchromatins, der aktiven Sequenzabschnitte, derzeit kritisch diskutiert wird, wird neben der Demethylierung der DNA selbst, die Azetylierung der aminoterminalen Abschnitte der Histone als Voraussetzung für die lockere Faltung der Histonabschnitte angesehen ( $\bullet$ Abb. 1)

Während Lysin-Azetylierungen zuverlässig mit einem permissiven Chromatinzustand assoziiert werden können, ist dies z.B. für die Methylierung von Lysinen nicht der Fall. Die Modifikation der Aminoterminalen ist aber nicht beschränkt auf Azetylierung, sondern kann durch Phosphorylierung, Ubiquitinierung und Methylierung weitere Änderungen erfahren.

Auch hier scheinen diese Veränderungen miteinander verwoben zu sein. Methylierungen können (mit 1, 2 oder 3 Methylgruppen) zusammen mit einem aktivierten Azetyl auftreten und sind z. B. in der trimethylierten Form an den Lysinen 4, 36 und 79 des Histons 3 (H3, K4, ME, A3, K36, ME oder H3K79 ME) mit transkriptionaler Aktivität assoziiert, während Methylierung an anderen Positionen (9, 20, 27) mit Gen-Inaktivierung assoziiert wurde. Dabei kommt womöglich der Anzahl der Methylgruppen eine wichtige Rolle zu, wobei das monomethylierte Lysin eine andere Regulationsebene darstellen kann als das trimethylierte. Nicht nur qualitative, sondern auch quantitative Effekte scheinen dabei von Bedeutung zu sein und es werden z.B. in der Region der Transcriptions-Start-Site (TSS) andere Histonmodifikationen beobachtet als in weiter 3'-gelegenen Sequenzabschnitten desselben Promotors.

Histon-Modifizierungen wie die Azetylierung oder Methylierung werden durch entgegengesetzt wirkende enzymatische Reaktionen gesteuert. Die Enzyme werden auch als „Schreiber“ (writer) bzw. „Auslöscher“ (eraser) bezeichnet. Dahinter verbirgt sich die Vorstellung, dass die komplexe Modifizierung von Histonen eine Art Chiffre darstellt, den sog. „Histone-Code“, der von einer Reihe von Proteinen gelesen wird. Diese „reader“ binden an bestimme Muster im Histone-Code und rekrutieren weitere Proteine, die an der Regulation der Genexpression beteiligt sind. Die Vielzahl der Histon-Modifikationen stellt einen hochkomplexen Mechanismus dar, der verschiedenste Reize in adaptive Veränderungen der Genexpression konsolidiert.

\section{MicroRNAs}

Nahm man noch bis vor Kurzem an, dass der überwiegende Teil des Genoms (d.h. die etwa 90\%, die keine Proteine kodieren) ohne Funktion sind (,junk“), geht man heute davon aus, das eben dieser ,junk“ in nicht kodierende RNA transkribiert wird, der verschiedenste regulatorische Funktionen zugeschrieben werden. MicroRNAs (miRNA) sind 20-22 Nukleotide lange, nicht kodierende RNAs, die im Nukleus als doppelsträngige RNA entstehen, dort durch Endonukleasen geschnitten werden und nach Export ins Zytosol durch die RNAse-Dicer zur reifen, einzelsträngigen miRNA prozessiert werden.

MiRNA nehmen eine besondere Rolle bei der posttranskriptionellen Regulation der Genexpression ein, da eine einzelne miRNA an über 100 verschiedene mRNAs binden kann, und umgekehrt eine mRNA durch verschiedene microRNAs moduliert werden kann. So können bereits durch Änderungen der Expression weniger miRNAs zelluläre Netzwerke moduliert werden. Die zelluläre Konzentration von miRNAs wird u.a. durch Anpassung der Transkription, Prozessierung und Degradation reguliert. Eine Dysregulation der miRNA-Expression wurde bei einer Reihe verschiedener Erkrankungen beobachtet und v.a. auf eine veränderte DNA Methylierung von miRNA-Promotorregionen zurückgeführt [39].

MiRNAs binden an komplementäre Sequenzen der 3' untranslatierten Region von miRNAs und stellen eine Verbindung mit dem RNA-induzierten silencing complex (RISC) her, wodurch die Translation gehemmt und die gebundene mRNA abgebaut wird. Mittlerweile wurden mehr als 2500 miRNAs beim Menschen identifiziert (www.mirbase.org), wovon etwa die Hälfte im ZNS exprimiert wird und u.a. Prozesse wie Neurogenese, Differenzierung, Neuroplastizität und Synaptogenese reguliert [40].

MiRNAs können physiologische Lern- und Gedächtnisprozesse regulieren, indem sie die Expression von dafür notwendigen Proteinen regulieren [41] ( $\bullet$ Abb.2). Änderungen in der Expression verschiedener miRNAs sind assoziiert mit Alterungsvorgängen und neurodegenerativen Erkrankungen, u.a. der Alzheimer-Erkrankung [42].

Interessanterweise ließ sich im Hirngewebe von AlzheimerPatienten eine verminderte Expression von verschiedenen miRNAs nachweisen, die die APP-Expression, das APP Splicing 


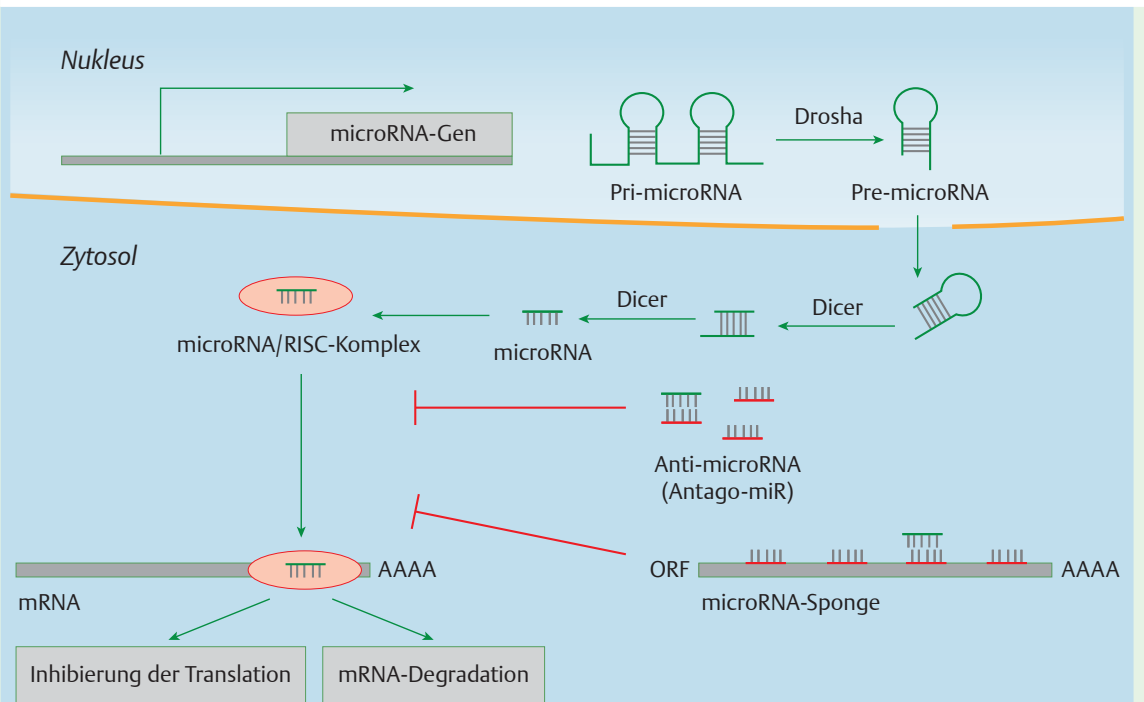

Abb.2 Gene für miRNA werden durch RNA-Polymerase zu primärer miRNA (pri-miRNA) transkribiert. Die pri-miRNA wird im Kern durch das Enzym Drosha zur pre-miRNA geschnitten und anschließend ins Zytosol exportiert, wo sie durch das Enzym Dicer zur doppelsträngigen miRNA prozessiert wird. Der Doppelstrang wird anschließend durch Dicer und einen Kofaktor TRBP zu Einzelsträngen entwunden, von denen der sogenannte guide-Strang an komplementäre Sequenzen seiner Ziel-mRNA bindet. Je nach Grad der Komplementarität wird dann entweder die Translation inhibiert oder die mRNA degradiert. Antagomirs sind Oligonukleotide, die komplementär zur Sequenz der endogenen miRNA sind, an diese binden und damit die Bindung der miRNA an ihre Ziel-mRNA inhibieren. miRNA sponges haben mehrere zu endogener miRNA komplementäre Sequenzfolgen und binden somit ebenfalls miRNAs, wodurch diese inhibiert werden.

oder die Expression von Schlüsselenzymen der Amyloid-betaProduktion regulieren [43]. Amyloid-beta wird durch die beiden Enzyme Beta-Secretase (BACE) und Gamma-Sekretase (Presenilin-Komplex) aus APP freigesetzt. Unter anderem konnte eine Korrelation von verminderter miRNA-29 und miRNA-107 und einer erhöhten BACE-1-Protein- bzw. BACE-1-mRNA-Expression in post mortem Alzheimer-Gewebe nachgewiesen werden [44]. Es gibt 3 Isoformen von APP, die durch alternatives Splicing entstehen und durch eine unterschiedlich hohe Rate der Amyloidbeta-Sekretion gekennzeichnet sind [45]. In Zellkulturexperimenten führte die Expression von miRNA-124 zu einer Reduktion von Exon-7- und -8-Splice-Varianten [46]. Interessanterweise ist die Konzentration von miRNA-124 in post mortem AD-Gehirngewebe verringert und APP-Isoformen, die Exon 7 und 8 enthalten, sind im Vergleich zu APP aus Kontrollgehirngewebe erhöht [47].

Das Mikrotubulus-assoziierte Protein tau (MAPT) aggregiert bei der Alzheimer-Erkrankung in einer pathologisch hyperphosphorylierten Form zu intrazellulären neurofibrillären Bündeln (NFTs) [48]. Eine Überexpression von MAPT kann die Aggregation des Proteins zu NFTs verstärken. In Zellkulturexperimenten zeigten Dickson et al., dass miRNA-34a die Expression von MAPT regulieren kann [49]. MAPT-phosphorylierende Kinasen werden in ihrer Expression und Aktivität ebenfalls durch verschiedene microRNAs reguliert (u.a. die cdk5-aktivierende miRNA-26b) und könnten dadurch MAPT-Pathologie bei Alzheimer beeinflussen.

Die miRNA-34c-Expression ist altersabhängig im Hippocampus von alternden Mäusen erhöht [50]. Interessanterweise finden sich auch im humanen post mortem Hippocampus bei Alzheimer-Patienten im Vergleich zu Gesunden erhöhte miRNA34Konzentrationen [51]. Wir konnten im Alzheimer-Mausmodell zeigen, dass eine erhöhte Expression von hippocampaler miRNA34c zu Defiziten im Lernen und Gedächtnis führt [50]. Umgekehrt konnten Defizite im assoziativen Lernen, die mit zunehmendem Alter oder in transgenen Alzheimer-Mausmodellen auftraten, durch die Applikation eines miRNA34-Seed-Inhibitors gebessert werden. Ein möglicher Mechanismus, über den eine pathologisch erhöhte Konzentration von miRNA34c zu Gedächtnisdefiziten führen kann, ist die miRNA34c-regulierte Expression von Sirtuin-1, dessen Verlust mit schlechterer Lern- und Gedächtnisleistung einhergeht [52].
Im Gegensatz zur erhöhten Expression von miRNA34c bei der Alzheimer-Krankheit wurden bei Parkinson-Patienten verringerte miRNA-34b/c-Konzentrationen im Gehirngewebe nachgewiesen, die gemeinsam mit einer Verminderung von Parkinson-assoziierten Genen DJ1 und Parkin auftraten [53]. DJ1- und Parkinmutationen führen zu einer autosomal-rezessiv vererbten familiären Parkinson-Erkrankung. DJ1 fungiert u.a. als ein Chaperon, das Zellen gegen oxidativen Stress schützt, Parkin ist eine neuroprotektive Ubiquitin-Ligase, die u. a. Mitochondrien vor Mitophagie unter Stressbedingungen schützt und das im Komplex mit DJ1 den proteasomalen Abbau von fehlgefalteten Proteinen fördert [54]. MiRNAs, die die Expression von SNCA regulieren, sind u. a. microRNA-7 und -153, die an die 3' untranslatierte Region der SNCA mRNA binden und im transgenen Parkinson-Mausmodell zu einer verminderten Expression von alpha-Synuklein führen [55]. Diese Befunde könnten möglicherweise für eine therapeutische Anwendung von Bedeutung sein, auch wenn noch unklar ist, ob auch bei Parkinson-Patienten eine Dysregulation dieser miRNAs vorliegt.

\section{Epigenetische Veränderungen bei neurodegenerativen Erkrankungen $\nabla$}

\section{Erkrankungen mit eindeutig epigenetischen Mechanismen}

Bei einer Reihe von neurologischen Erkrankungen finden sich Mutationen in Genen, die direkt und unmittelbar in die epigenetische Regulation eingreifen, dies sind u.a. das Rett-Syndrom (Mutationen und Duplikationen des methyl-CPG-bindenden Proteins; MECP2), das Rubinstein-Taybi-Syndrom, das Coffin-LowrySyndrom und das ATRX-Syndrom, die kürzlich im Detail beschrieben worden sind [6]. Andere Erkrankungen wie die Huntington'sche Erkrankung und die spinozerebelläre Ataxie Typ 3 sind durch Mutationen in ubiquitär exprimierten Genen gekennzeichnet, die in einem zell- und gewebespezifischen Zusammenhang mit spezifischen Komponenten der epigenetischen Regulation wechselwirken: Ataxin-3 und Huntington sind jeweils Bestandteile unterschiedlicher Transkriptionsrepressorkomplexe. Eine dritte Gruppe von Erkrankungen ist durch epigenetische Veränderungen in strukturell veränderter genetischer Umgebung gekennzeichnet. Das fragile X-Syndrom, eine der häufigsten For- 
men angeborener Minderbegabung, welche durch expandierte CGG-Wiederholungen hervorgerufen wird und die FriedreichAtaxie, die durch expandierte GAA-Wiederholungen hervorgerufen wird, sind jeweils durch deutliche Hypermethylierung von CPG-Motiven in den Promotorregionen der mutierten Gene gekennzeichnet [7].

Bei anderen ZNS-Erkrankungen mit starker metabolischer Komponente wie z.B. Drogenabhängigkeit, Alkoholismus und Anorexie wurden globale und genspezifische Veränderungen der Methylierung nachgewiesen [8]. Auch bei Patienten mit sporadischen Erkrankungen des Nervensystems sind bereits erste Analysen des gesamten Epigenoms durchgeführt worden $[9,10]$.

\section{Morbus Alzheimer}

Der Morbus Alzheimer (Alzheimer's disease, $\mathrm{AD}$ ) ist die häufigste neurodegenerative Erkrankung und histopathologisch durch extrazelluläre Ablagerung amyloider Plaques und intrazelluläre Aggregation des Mikrotubulus-assoziierten Proteins Tau charakterisiert. Amyloide Plaques entstehen durch Spaltung des Amyloid-Vorläufer-Proteins APP und Freisetzung des Amyloid-betaPeptids, das zu fibrillären Plaques aggregiert. Die genetische Form von Morbus Alzheimer ist auf Mutationen in Genen zurückzuführen, welche die Prozessierung des Amyloid-beta-VorläuferProteins (amyloid precursor protein, APP) beeinflussen, macht aber nur 2-5\% aller Fälle aus [11]. Dabei sind beeinträchtigende Gedächtnisfunktionen bereits in vergleichsweise jungen Personen zu beobachten. Die weitaus häufigere sporadische Form, welche die restlichen 95-98\% aller Fälle ausmacht, zeichnet sich auch durch amyloide Plaques aus, tritt aber erst in höherem Lebensalter auf. Welche Bedeutung diesen amyloiden Plaques tatsächlich zukommen könnte wird derzeit sehr kontrovers diskutiert. Neben den Plaques sind die neurofibrillären Bündel („tangles“) ein weiteres pathologisches Merkmal der AlzheimerKrankheit. Dabei handelt es sich um intrazelluläre Zusammenlagerungen von abnormal phosphoryliertem Mikrotubulus-assoziiertem TAU-Protein (MAPT), welches eine wichtige Funktion bei intrazellulären Transportprozessen hat [12]. Mutationen im tau-Gen wurden bisher zwar nicht mit Alzheimer in Verbindung gebracht, führen aber zur frontotemporalen Demenz (FTD) [13]. Geleitet durch die genetischen Befunde hat sich die AlzheimerForschung in der Vergangenheit im Wesentlichen auf die Amyloidpathologie konzentriert. Leider sind die entsprechenden klinischen Versuche gescheitert und wirksame therapeutische Ansätze sind bisher nicht entstanden. Ganz zweifellos muss die sporadische Form von Alzheimer (ebenso wie die sporadische Parkinson-Erkrankung) als eine komplexe Erkrankung infolge variabler Interaktionen von genetischem Risiko und Umweltfaktoren angesehen werden.

Da epigenetische Mechanismen als Schlüsselprozesse GenomUmwelt-Interaktionen regulieren und jüngste Daten darauf hinweisen, dass die Epigenetik eine wichtige Funktion bei Lern- und Gedächtnisprozessen hat, entwickelt sich ein neues, dynamisches Forschungsgebiet, um die Epigenetik bei Alzheimer zu untersuchen. Dass die Azetylierung von Histonen Einfluss auf Lernprozesse haben könnte, wurde erstmals bereits 1979 gezeigt, geriet dann aber in Vergessenheit [14]. Spätere Arbeiten zeigten dann, dass Gedächtniskonsolidierung im Tiermodell mit einer veränderten Histonazetylierung und HAT-Aktivität in den relevanten Hirngebieten einhergeht [15]. Auf funktioneller Ebene konnte durch genetische Experimente gezeigt werden, dass Tiere, in denen HATs im Gehirn ausgeschaltet sind, schlechter lernen und dieses Lerndefizit konnte durch die Gabe von HDAC-Inhibi- toren kompensiert werden [16]. Überexpression von HDA1c vermindert die Dichte dendritischer Buttons und die Anzahl von Synapsen in Mäusen während einer Unterdrückung von HDA2 zu einer gesteigerten Anzahl von Synapsen und verbesserten Gedächtnisfunktionen führt. Naheliegend wäre also die Annahme einer Störung der Histonazetylierung und De-Azetylierung in Erkrankungen mit Gedächtnisstörungen wie eben der AlzheimerKrankheit.

Das therapeutische Potenzial von veränderter Histonazetylierung bei AD wurde erstmals im sogenannten CK-p25-Tiermodell untersucht. P25 ist eine pathologische Untereinheit der zyklinabhängigen Kinase 5 (CDK5) die z. B. in Hirnen von Alzheimer-Patienten verstärkt nachzuweisen ist. Die Überexpression von P25 im Vorderhirn der Maus führt zu massiver Neurodegeneration sowie zur Ausbildung von neurofibrillären Bündeln, erhöhtem Gehalt an $A \beta$-Peptiden und Gedächtnisverlust. Die Gabe des HDAC-Inhibitors Sodiumbutyrate (tägliche intraperitoneale Injektion für 4 Wochen) konnte das Lernvermögen in CK-p25-Tieren selbst dann wiederherstellen, wenn die pathologischen Veränderungen bereits weit fortgeschritten waren [17]. Auf molekulare Ebenen konnten nicht nur protektive, sondern auch regenerative Prozesse beobachtet werden, wie z.B. die verbesserte Verknüpfung von Nervenzellen. Auch konnte z.B. gezeigt werden, dass der HDAC-Inhibitor Trichostatin A (TSA) physiologische Histone-H4-Azetylierung in APPPS1-Tieren, einem Tiermodell für Amyloidpathologie, wiederherstellt und das Lernen deutlich verbessert [18]. Ähnliche Beobachtungen wurden in einem anderen Tiermodell, den sogenannten Tg2576-Mäusen, gemacht. Eine 5wöchige Applikation (tägliche intraperitoneale Injektion) des HDAC-Inhibitors Phenylbutyrat erhöhte die Histone-H4-Azetylierung, verbesserte das Lernen und die synaptische Plastizität [19]. Der relativ selektive HDAC-Inhibitor Suberoylanilide-Hyrdxamate (SAHA, Vorinostat; HDAC1, HDAC2, HDAC3 und HDAC8) konnte durch tägliche intraperitoneale Injektion für 3 Wochen das Lernvermögen in APPPS1-delta9-Tieren verbessern [20]. Scheinbar kann die Gabe von HDAC-Inhibitoren kognitive Funktionen im Tiermodell für Alzheimer auch verbessern, wenn die Tiere sich bereits in einem fortgeschrittenen Stadium befinden [21]. Außerdem konnten HDAC-Inhibitoren Lernen in Mausmodellen für altersbedingten Gedächtnisverlust verbessern [22]. Eine Studie hat kürzlich den HDAC1-Inhibitor MS-275 (Entinostat) im Mausmodell für Amyloidpathologie untersucht. MS-275 konnte kognitive Funktionen wiederherstellen, aber auch die Anzahl der amyloiden Plaques sowie neuroinflammatorische Prozesse reduzieren [23]. Diese Ergebnisse deuten darauf hin, dass HDAC-Inhibitoren einerseits über die Wiederherstellung physiologischer Histonazetylierung die Genexpressionsmuster normalisieren, gleichzeitig aber vermutlich auch entzündliche Prozesse regulieren [24].

Die Wirkweise von HDAC-Inhibitoren ist also vielfältig, was einerseits Vorteile bieten kann, andererseits aber auch die Gefahr unerwünschter Arzneimittelwirkungen (UAW; „off target effects“) erhöht. Die Wirkweise der 11-HDAC-Proteine im Gehirn war bis vor 5 Jahren weitgehend unbekannt. Diese 11 HDACs werden in 2 Klassen eingeteilt: HDAC1, 2, 3 und 8 sind meist im Zellkern lokalisiert und regulieren dort die Genexpression. Die Klasse-II-HDACs ist auch im Zytoplasma und anderen zellulären Kompartimenten zu finden und reguliert nicht nur die Genexpression, sondern auch andere Prozesse [17].

Deletion von HDAC2 in den Mäusen führt zu einem besseren Lernvermögen und erhöhter Histon-H4-Azetylierung und wirkt Gedächtnisverlust im Modell für Amyloidpathologie entgegen 
[25]. Ähnliche Befunde wurden für HDAC3 beobachtet. Auf der anderen Seite konnte gezeigt werden, dass eine Reduzierung des HDAC5-Gehalts nachteilig wirkt, also eine Inhibierung in jedem Fall vermieden werden sollte. HDAC6 reguliert v.a. die Azetylierung von Mikrotubuli und Proteinen wie dem Hitzeschockprotein HSP-90. Ähnlich wie für HDAC2, konnte gezeigt werden, dass die Reduzierung des HDAC6-Gehalts im Tiermodell für amyloide Pathologie Lernvermögen verbessert, aber keinen Einfluss auf die Ausbildung von amyloiden Plaques hat. Da auch das TAU-Protein azetyliert wird und dieser Vorgang Einfluss auf die Alzheimer-Pathologie hat, könnte HDAC6-TAU-Pathologie beeinflussen. Erste Hinweise darauf stammen aus Drosophila-Modellen. Hier konnte gezeigt werden, dass TAU-Pathologie zu einer Destabilisierung von Mikrotubuli führt, was durch Deletion von HDAC6 verhindert werden konnte [26]. Diese Ergebnisse sind aber auch deshalb von Bedeutung, da HDAC-Inhibitoren wie SAHA (Vorinostat) Klasse-I-HDACs und -HDAC6 inhibieren und bereits für andere Indikationen zugelassen sind [27].

Im Gegensatz zu den HDAC-Proteinen ist relativ wenig über die Funktion der HATs im adulten Gehirn bekannt. Gut untersucht ist lediglich das bereits erwähnte CBP-Protein, welches essenziell für kognitive Funktionen ist und bei Ausfall zum Rubinstein-Taybi-Syndrom führt. Zu den anderen 17-HAT-Proteinen ist im Zusammenhang mit kognitiven Prozessen nur wenig bekannt; nur einzelne HAT-Agonisten wurden bereits in Tiermodellen untersucht und verbessern ebenso wie HDAC-Inhibitoren die Gedächtnisleistung [28].

Diese ersten Ergebnisse sind vielversprechend und epigenetische therapeutische Ansätze wie der Einsatz von HDAC-Inhibitoren werden mittlerweile von einer Reihe von Pharmaunternehmen verfolgt. Ob sich die Daten aus Tiermodellen auf den Mensch übertragen lassen, ist derzeit noch nicht abzusehen. Eine große Hoffnung ist, dass neben der Wiederherstellung von physiologischer Genexpression, HDAC-Inhibitoren auch inflammatorische Prozesse reduzieren und auch dann wirken könnten, wenn die Krankheit bereits fortgeschritten ist.

Neben den HDACs bieten auch andere epigenetische Regulatoren neue therapeutische Ziele: Histonmethyltransferasen (HMT) und Histonedemethylasen (HDMs) regulieren die Methylierung von Histonen, scheinen spezifischer als HDACs zu agieren und ebenfalls von Bedeutung für Lernprozesse zu sein [29].

\section{Morbus Parkinson}

Der Morbus Parkinson (MP; Parkinson's disease, PD) ist nach der Alzheimer'schen Erkrankung die zweithäufigste neurodegenerative Krankheit des Menschen und betrifft etwa 2\% der Bevölkerung über 65 Jahre. Aktuelle Schätzungen der „Parkinson's Disease Foundation“, gehen von 1 Million Betroffenen in den USA und mehr als 4 Millionen Menschen weltweit aus; in Anbetracht der demografischen Entwicklung wird für 2020 mit etwa 8 Millionen PD-Patienten gerechnet. Ganz ähnlich wie bei Alzheimer ist die überwiegende Mehrzahl aller Fälle sporadisch (>95\%) und trotz intensiver Forschung ist die Ätiologie unklar.

Die besondere Bedeutung von alpha-Synuklein-Gen (SNCA) und -Protein (a-SYN) wurde 1997 deutlich, als eine dominante Mutation im SNCA-Gen identifiziert wurde, die ein familiäres Parkinson-Syndrom mit hoher Penetranz auslöst. Der SNCA-Gen-Lokus wurde ursprünglich in einer großen Familie mit dominant vererbtem MP und Lewy-Körperchen-Pathologie kartiert; 2 weitere Punktmutationen mit hoher Penetranz wurden in großen Familien identifiziert [30]. In der Folge konnte gezeigt werden, dass das a-SYN-Protein Hauptbestandteil auch der charakteristischen
Lewy-Körper ist, die in den Nervenzellen der Patienten mit sporadischer Parkinson-Krankheit gefunden wurden [31]. Wie der Name impliziert, wurde a-SYN initial als synaptisches und nukleäres Protein identifiziert. Trotz intensiver Studien ist die genaue physiologische Rolle von a-SYN noch nicht klar definiert: möglicherweise spielt a-SYN eine Rolle bei der Aufrechterhaltung des synaptischen Vesikelpools [32]. Bereits ein geringer Anstieg der a-SYN-Menge scheint ausreichend, um synaptische Dysfunktion und Degeneration zu induzieren [33].

Das die Menge des a-SYN-Proteins und damit die Expressionsstärke des SNCA-Gens für die Pathogenese des MP von entscheidender Bedeutung sind, zeigen die (ebenfalls dominant vererbten) Duplikationen und Triplikationen des SNCA-Gens, die zu einem MP führen [34]. Gendosiseffekte und die Regulation der SNCA-Expression spielen eine wichtige Rolle auch für den sporadischen MP: mehrere Studien belegen Assoziationen von verschiedenen genetischen Varianten (single nucleotide polymorphisms; SNP) in der Promotorregion des SNCA-Gens bei sporadischem MP [PD Gene Database].

Die Expressionsstärke eines Gens wird jedoch nicht nur durch Veränderungen der kodierenden (Promotor-)Sequenz, sondern eben auch durch mehrere Ebenen epigenetischer Regulation bestimmt [35]. Tatsächlich beeinflusst der Methylierungsstatus des Intron1 des SNCA-Gens die Transkription und es gibt Hinweise aus der Untersuchung von DNA aus Gehirnen von MP-Patienten, dass im Vergleich zu Gesunden dieser transkriptionell aktive Bereich des SNCA-Gens weniger stark methyliert ist, was die Bildung des a-SYN-Proteins begünstigt [36]. Diese epigenetische Regulation der Proteinmenge könnte das Phänomen erklären, dass Verwandte von Patienten mit MP ein höheres Erkrankungsrisiko haben, obwohl MP üblicherweise nicht nach den Mendel'schen Regeln vererbt wird.

Epigenetische Veränderungen, die im ZNS anhand der Ergebnisse, die an post mortem Material gewonnen wurden, vermutet werden, könnten aber auch durch Medikamente beeinflusst sein: in Modellsystemen der Parkinson-Erkrankung wurde gezeigt, dass Dopamindepletion zu einer Verminderung von trimethyliertem H3K4 im Striatum führt, während die Dauerbehandlung mit Levodopa zu einer De-Azetylierung von Histon H4 an den Positionen 5, 8, 12 und 16 führte [37]. Studien an Drosophila zeigten, dass $\alpha$-SYN direkt an Histone binden und den Gehalt von azetyliertem Histon H3 vermindern kann [38]. Wir konnten zeigen, dass das Gen für Cytochrom P450 2E1 in Gehirnen von MPPatienten hypomethyliert ist und dabei auch belegen, dass dieser Effekt die Menge des Proteins reguliert [9].

\section{Epigenetische Perspektiven in Diagnostik und Therapie $\nabla$}

Die Tatsache, dass Histonmodifizierungen in Mausmodellen und in post mortem Gewebeproben beobachtet werden, wirft die Frage auf, inwieweit solche Modifikationen als Biomarker dienen könnten. Bei zytologischen Untersuchungen des Liquors konnten zwar Histone nachgewiesen werden, im Zusammenhang mit AD oder MP gibt es hierzu aber bisher keine Daten. Lymphozyten stellen eine andere mögliche Quelle dar, um Histonmodifizierungen zu messen. Auch hier gibt es bisher keine Daten. Ein generelles Problem solcher Ansätze liegt in der Ungewissheit, ob Prozesse, die im Gehirn ablaufen, im Blut abgebildet werden. Tatsächlich gibt es mittlerweile Methoden der molekularen Bildgebung, die z. B. in lebenden Zellen die Azetylierung von H4 am Lysine 12, 
einer bei für Alzheimer wichtigen Histonmodifizierung messen können [56]. Die Umsetzung solcher Ansätze in klinisch relevante bildgebende Verfahren scheint aber nicht in naher Zukunft realisierbar.

Epigenetische Prozesse wie die Histonazetylierung beeinflussen auch die Expression von nicht kodierenden RNAs wie z.B. der miRNAs, die aussichtsreichere Biomarker für Hirnerkrankungen darstellen [57].

HDAC-Inhibitoren (HDACi) könnten neue therapeutische Werkzeuge darstellen. Für die Tumortherapie wurden bereits unterschiedliche Klassen von HDACi entwickelt und in verschiedenen Phase-I-III-Studien klinisch getestet [58]. Bislang erhielten nur der pan-Klasse-I(+II)-Inhibitor Vorinostat sowie Romidepsin, das v.a. HDACs 1 und 2 inhibiert, eine Zulassung für die Therapie therapierefraktärer kutaner T-Zell-Lymphome. Wichtig für das Design eines HDACis zur Therapie neurodegenerativer Erkrankungen sind die Verträglichkeit, die Penetrationsfähigkeit durch die Blut-Hirn-Schranke sowie die Selektivität gegenüber den verschiedenen HDAC-Klassen. Von den in Phase II und III getesteten HDAC-Inhibitoren wurde eine Blut-Hirn-Schranken-Permeabilität nur für die HDAC1-Inhibitoren Etinostat (MS-275) und Vorinostat berichtet. Etinostat konnte in einem Alzheimer-Mausmodell Inflammation und die Ablagerung amyloider Plaques reduzieren [59]. In einem Mausmodell für traumatische Hirnschädigung zeigten mit Etinostat therapierte Tiere eine verbesserte kognitive Leistung [60]. Vorinostat inhibiert v. a. die Histondeazetylasen HDAC1, 2, 3 und HDAC6, die alle im Zusammenhang mit der Pathogenese der Alzheimer-Erkrankung in Zusammenhang gebracht werden. Im Gegensatz dazu führt die Inhibierung von Klasse-II-HDACis zu einer deutlichen Verschlechterung der Gedächtnisleistung, sodass HDACi mit einer Aktivität gegenüber HDAC5, vermieden werden sollten [61]. Um das Nebenwirkungsprofil gering zu halten, wäre die Entwicklung möglichst spezifischer HDAC-Inhibitoren wünschenswert.

Vorinostat, das in verschiedenen Alzheimer-Mausmodellen positiv getestet wurde, war in klinischen Studien v.a. durch eine Verlängerung der QTc-Zeit, Fatigue, einer Suppression der Hämatopoese, Übelkeit, Erbrechen und Diarrhö in seiner Dosierung und Dauer der Anwendung limitiert. Sicherheitsdaten zur kontinuierlichen Anwendung und v.a. zur Therapie bei älteren Patienten wurden bislang nicht erhoben.

Interessanterweise weist das Antikonvulsivum Valproat in einer gut verträglichen und therapeutisch genutzten Dosierung eine schwache HDAC-inhibierende Wirkung auf [60]. In onkologischen Studien an hämatologischen und soliden Tumoren konnte eine biologische Wirksamkeit auf Genexpression und eine klinische Wirkung von Valproat auf den Krankheitsverlauf demonstriert werden. Allerdings trat in einigen dieser Studien Neurotoxizität als dosislimitierender Faktor auf. Im Alzheimer-Mausmodell konnten Kilgore et al. (2010) zeigen, dass eine Therapie mit Valproat zu einer verbesserten Gedächtnisleistung führt [63]. Die Interpretation dieses Befunds ist allerdings schwierig, da Valproat über unterschiedliche potenzielle Wirkmechanismen bei der Alzheimer-Erkrankung ansetzen könnte: über eine Inhibierung der Histondeazetylaseaktivität, aber auch über eine Inhibierung der pathologischen Hyperphosphorylierung von Tau durch GSK3b-Kinase oder über eine Stabilisierung des Membranpotenzials. Eine multizentrische, placebokontrollierte und verblindete Studie mit Valproat an 313 Patienten mit moderater AlzheimerDemenz zeigte keinen positiven Effekt auf die Geschwindigkeit, mit der kognitive oder funktionelle Defizite im Krankheitsverlauf zunahmen [64]. Dies deckt sich auch mit der klinischen Erfah- rung mit Valproat als Antikonvulsivum. Das negative Studienergebnis könnte jedoch dem späten Therapiebeginn geschuldet sein, da zu diesem Zeitpunkt bereits ein großes Ausmaß irreversibler neuronaler Schädigung erfolgt ist und möglicherweise eine nicht mehr aufhaltbare neurotoxische Kaskade begonnen hat. MiRNA können als therapeutisches Ziel entweder durch sogenannte "miRNA mimics“ oder als "anti-miRNAs“, d.h. RNA-Sequenzen, die einer miRNA entsprechen und die Proteinexpression entsprechend ihrer Zielsequenz inhibieren, adressiert werden.

Es gibt verschiedene Ansätze, um miRNAs zu inhibieren. Antagomirs sind cholesterolgekoppelte RNA-Sequenzen, die komplementär zur miRNA sind, die gehemmt werden soll [65]. Dabei hilft das Cholesterolmolekül, den Transfer der RNA über die Zellmembran zu erleichtern. Für eine therapeutische Anwendung im Bereich neurodegenerativer Erkrankungen lassen sich Antagomirs allerdings nicht nutzen, da sie nicht die Blut-Hirn-Schranke penetrieren können. Synthetische mRNA „sponges“ werden mit komplementären Sequenzen für die Ziel-miRNA hergestellt und verringern die zur Verfügung stehende Menge ungebundener, biologisch aktiver miRNA [66].

Eine miRNA-gerichtete Therapie birgt einige Probleme, u.a. die fehlende Spezifität einer miRNA und die unklare Halbwertszeit in vivo, bedingt durch mögliche hepatorenale Elimination oder Degradation durch Nukleasen. Weitere Schwierigkeiten sind der Transfer über die Blut-Hirn-Schranke, die Schwierigkeit, spezifisch Zielzellen eines bestimmten Gewebes zu adressieren und die effiziente Aufnahme von Mimics in diese Zielzellen sowie die Freisetzung aus dem Endolysosom [67]. Verschiedene experimentelle Strategien wurden entwickelt, um diese Probleme zu umgehen. RNA kann zum Schutz vor Nukleasen und zur besseren zellulären Aufnahme in Lipopartikel verpackt werden. Eine Konjugation mit positiv geladenen Trägermolekülen kann dabei die Aufnahme über negativ geladene Zellmembranen weiter verbessern. Um die Substanzen möglichst gewebsspezifisch zu adressieren, wurden v.a. die Kopplung an Aptamere, Antikörper oder Liganden experimentell getestet. Eine Kombination aus Verpackung in Nanopartikel und Zielzelladressierung mit einem Liganden wurde von Alvarez-Erviti et al. 2011 für siRNA beschrieben. Diese wurde mittels Elektroporation in Exosomen verpackt, die einen Azetylcholinrezeptor-Liganden an der Oberfläche trugen. Diese Methode bietet zum einen Schutz vor Nukleasen, zum anderen aber auch neben der Gewebsspezifität den Vorteil, dass Exosomen effizient die Blut-Hirn-Schranke penetrieren können. In Mausexperimenten konnte dadurch eine verringerte Expression der Beta-Sekretase in Neuronen erzielt werden [68]. Eine Übertragung dieses Prinzips auf miRNA gerichtete Therapeutika ist denkbar. Eine weitere Option ist die virale Expression von miRNAs unter einem gewebsspezifischen Promotor.

\section{Fazit}

\section{$\nabla$}

Die Analyse epigenetischer Prozesse stellt einen neuen, vielversprechenden Ansatzpunkt zur Stratifizierung von Patienten und zur Entwicklung neuer Therapieverfahren dar. Neben dem besseren Verständnis epigenetische Mechanismen bei ZNS-Erkrankungen, wird die Translation solcher Ergebnisse aus Grundlagenund präklinischer Forschung in konkrete Anwendungen eine der größten Herausforderungen für die Zukunft darstellen. 


\section{Interessenkonflikt}

$\nabla$

Die Autoren geben an, dass kein Interessenkonflikt besteht.

\section{Literatur}

1 Suzuki MM, Bird A. DNA methylation landscapes: provocative insights from epigenomics. Nat Rev Genet 2008; 9: 465-476

2 Dias BG, Ressler KJ. Parental olfactory experience influences behavior and neural structure in subsequent generations. Nat Neurosci 2014; 17: 89-96

3 Siegmund KD, Connor CM, Campan M et al. DNA methylation in the human cerebral cortex is dynamically regulated throughout the life span and involves differentiated neurons. PLoS ONE 2007; 2: e895

4 Heijmans BT, Tobi EW, Stein $A D$ et al. Persistent epigenetic differences associated with prenatal exposure to famine in humans. PNAS U S A 2008; 105: 17046 - 17049

5 Murgatroyd C, Patchev $A V, W u Y$ et al. Dynamic DNA methylation programs persistent adverse effects of early-life stress. Nat Neurosci 2009; 12: $1559-1566$

6 Urdinguio RG, Sanchez-Mut JV, Esteller M. Epigenetic mechanisms in neurological diseases: genes, syndromes, and therapies. The Lancet Neurology 2009; 8: 1056-1072

7 Hornstra IK, Nelson DL, Warren ST et al. High resolution methylation analysis of the FMR1 gene trinucleotide repeat region in fragile X syndrome. Hum Mol Genet 1993; 2: 1659-1665

8 Bönsch $D$, Lenz B, Fiszer $R$ et al. Lowered DNA methyltransferase (DNMT-3b) mRNA expression is associated with genomic DNA hypermethylation in patients with chronic alcoholism. J Neural Transm 2006; 113: $1299-1304$

9 Kaut 0 , Schmitt I, Wüllner U. Genome-scale methylation analysis of Parkinson's disease patients' brains reveals DNA hypomethylation and increased mRNA expression of cytochrome P450 2E1. Neurogenetics 2012; 13: 87-91

10 Yu L, Chibnik LB, Srivastava GP et al. Association of Brain DNA Methylation in SORL1, ABCA7, HLA-DRB5, SLC24A4, and BIN1 With Pathological Diagnosis of Alzheimer Disease. JAMA Neurol 2014: Nov 3; Doi: 10.1001/jamaneurol.2014.3049

11 Haass C, Selkoe DJ. Soluble protein oligomers in neurodegeneration: lessons from the Alzheimer's amyloid beta-peptide. Nat Rev Mol Cell Biol 2007; 8: $112-116$

12 Schneider A, Mandelkow E. Tau-based treatment strategies in neurodegenerative diseases. Neurotherapeutics 2008; 5: 443-457.13

13 Höglinger GU, Melhem NM, Dickson DW et al. Identification of common variants influencing risk of the tauopathy progressive supranuclear palsy. Nat Genet 2011; 43: 699-705

14 Schmitt M, Matthies H. Biochemical studies on histones of the central nervous system. III. Incorporation of [14C]-acetate into the histones of different rat brain regions during a learning experiment. Acta Biol Med Ger 1979; 38: 683-689

15 Swank MW, Sweatt JD. Increased Histone Acetyltransferase and Lysine Acetyltransferase Activity and Biphasic Activation of the ERK/RSK Cascade in Insular Cortex During Novel Taste Learning. J Neurosci 2001; 21: $3383-3391$

16 Alarcon JM, Malleret G, Touzani K et al. Chromatin acetylation, memory, and LTP are impaired in $\mathrm{CBP}+/$ - mice: a model for the cognitive deficit in Rubinstein-Taybi syndrome and its amelioration. Neuron 2004; 42 : 947-959

17 Fischer $A$, Sananbenesi F, Wang $X$ et al. Recovery of learning \& memory after neuronal loss is associated with chromatin remodeling. Nature 2007; 447: 178 - 182

18 Francis YI, Fà $M$, Ashraf $H$ et al. Dysregulation of histone acetylation in the APP/PS1 mouse model of Alzheimer's disease. J Alzheimers Dis 2009; 18: 131 - 139

19 Ricobaraza A, Cuadrado-Tejedor M, Pérez-Mediavilla A et al. Phenylbutyrate ameliorates cognitive deficit and reduces tau pathology in an Alzheimer's disease mouse model. Neuropsychopharmacology 2009; 34: $1721-1732$

20 Kilgore M, Miller CA, Fass DM et al. Inhibitors of class 1 histone deacetylases reverse contextual memory deficits in a mouse model of Alzheimer's disease. Neuropsychopharmacology 2010; 35: 870-880

21 Govindarajan N, Agis-Balboa C, Walter J et al. Sodium Butyrate Improves Memory Function in an Alzheimer's Disease Mouse Model When Administered at an Advanced Stage of Disease Progression. J Alzheimers Dis 2011; 24: 1-11
22 Fontán-Lozano A, Romero-Granados R, Troncoso J et al. Histone deacetylase inhibitors improve learning consolidation in young and in KAinduced-neurodegeneration and SAMP-8-mutant mice. MolCell Neurosci 2008; 39: 193-201

23 Zhang ZY, Schluesener HJ. Oral administration of histone deacetylase inhibitor MS-275 ameliorates neuroinflammation and cerebral amyloidosis and improves behavior in a mouse model. J Neuropathol Exp Neurol 2013; 72: 178-185

24 Fischer A. Epigenetic memory: the Lamarckian brain. EMBO J 2014; 33: 945-967

25 Guan JS, Haggarty SJ, Giacometti E et al. HDAC2 negatively regulates memory formation and synaptic plasticity. Nature 2009; 459: 55-60

26 Xiong Y, Zhao K, Wu J et al. HDAC6 mutations rescue human tau-induced microtubule defects in Drosophila. Proc Natl Acad Sci U S A 2013; 110: 4604-4609

27 Walkinshaw DR, Yang XJ. Histone deacetylase inhibitors as novel anticancer therapeutics. Curr Oncol 2008; 15: 237-243

28 Chatterjee S, Mizar P, Cassel $R$ et al. A novel activator of CBP/p300 acetyltransferases promotes neurogenesis and extends memory duration in adult mice. J Neurosci 2013; 33: 10698-10712

29 Schaefer A, Sampath SC, Intrator A et al. Control of cognition and adaptive behavior by the GLP/G9a epigenetic suppressor complex. Neuron 2009; 64: 678-691

30 Polymeropoulos $M H$, Lavedan C, Leroy E et al. a-synuclein gene identified in families with Parkinson's disease. Science 1997; 276: 2045 2047

31 Spillantini MG, Schmidt ML, Lee VM et al. Alpha-synuclein in Lewy bodies. Nature 1997; 388: 839-840

32 Abeliovich A, Schmitz Y, Farinas I et al. Mice lacking alpha-synuclein display functional deficits in the nigrostriatal dopamine system. Neuron 2000; 25: 239-252

33 Nemani VM, Lu W, Berge $V$ et al. Increased expression of alpha-synuclein reduces neurotransmitter release by inhibiting synaptic vesicle reclustering after endocytosis. Neuron 2010; 65: 66-79

34 Singleton $A B$, Farrer $M$, Johnson $J$ et al. alpha-Synuclein locus triplication causes Parkinson's disease. Science 2003; 302: 841

35 Feinberg AP. Phenotypic plasticity and the epigenetics of human disease. Nature 2007; 447: 433-440

36 Jowead A, Schmitt I, Kaut $O$ et al. Methylation regulates expression of alpha-synuclein and is decreased in Parkinson's disease patients' brains. J Neurosci 2010; 30: 6355-6359

37 Nicholas AP, Lubin FD, Hallett PJ et al. Striatal histone modifications in models of levodopa-induced dyskinesia. J Neurochem 2008; 106: $486-494$

38 Chen L, Feany MB. Alpha-synuclein phosphorylation controls neurotoxicity and inclusion formation in a Drosophila model of Parkinson disease. Nat Neurosci 2005; 8: 657-663

39 Van den Hove DL, Kompotis K, Lardenoije R et al. Epigenetically regulated microRNAs in Alzheimer's disease. Neurobiol Aging 2014; 35: 731 745

40 Ziats MN, Rennert OM. Identification of differentially expressed microRNAs across the developing human brain. Mol Psychiatry 2014; 19: $848-852$

41 Gao J, Wang WY, Mao YW et al. A novel pathway regulates memory and plasticity via SIRT1 and miR-134. Nature 2010; 466: 1105-1109

42 Abe M, Bonini NM. MicroRNAs and neurodegeneration: role and impact. Trends Cell Biol 2013; 23: 30-36

43 Nunez-Iglesias J, Liu CC, Morgan TE et al. Joint genome-wide profiling of miRNA and mRNA expression in Alzheimer's disease cortex reveals altered miRNA regulation. PLoS One 2010; 5: e8898

44 Wang WX, Huang $\mathrm{Q}$ Hu Yet al. Patterns of microRNA expression in normal and early Alzheimer's disease human temporal cortex: white matter versus gray matter. Acta Neuropathol 2011; 121: $193-205$

45 Donev $R$, Newall A, Thome J et al. A role for SC35 and hnRNPA1 in the determination of amyloid precursor protein isoforms. Mol Psychiatry 2007; 12: $681-690$

46 Smith P, Al Hashimi A, Girard J et al. In vivo regulation of amyloid precursor protein neuronal splicing by microRNAs. J Neurochem 2011; 116: $240-247$

47 Rockenstein EM, McConlogue L, Tan $H$ et al. Levels and alternative splicing of amyloid beta protein precursor (APP) transcripts in brains of APP transgenic mice and humans with Alzheimer's disease. Biol Chem 1995; 270: $28257-28267$ 
48 Mandelkow EM, Mandelkow E. Biochemistry and cell biology of tau protein in neurofibrillary degeneration. Cold Spring Harb Perspect Med 2012; 2: a006247

49 Dickson JR, Kruse C, Montagna DR et al. Alternative polyadenylation and miR-34 family members regulate tau expression. J Neurochem 2013; 127: 739-749

50 Zovoilis A, Agbemenyah HY, Agis-Balboa RC et al. microRNA-34c is a novel target to treat dementias. EMBO J 2011; 30: 4299-4308

51 Müller M, Kuiperij HB, Claassen JA et al. MicroRNAs in Alzheimer's disease: differential expression in hippocampus and cell-free cerebrospinal fluid. Neurobiol Aging 2014; 35: 152 - 158

52 Michán S, Li Y, Chou MM et al. SIRT1 is essential for normal cognitive function and synaptic plasticity. J Neurosci 2010; 30: 9695-9707

53 Miñones-Moyano E, Porta S, Escaramís G et al. MicroRNA profiling of Parkinson's disease brains identifies early downregulation of miR34b/c which modulate mitochondrial function. Hum Mol Genet 2011; 20: $3067-3078$

54 Müller-Rischart AK, Pilsl A, Beaudette P et al. The E3 ligase parkin maintains mitochondrial integrity by increasing linear ubiquitination of NEMO. Mol Cell 2013; 49: 908-921

55 Junn E, Lee KW, Jeong BS et al. Repression of alpha-synuclein expression and toxicity by microRNA-7. Proc Natl Acad Sci U S A 2009; 106: $13052-13057$

56 Ito $T$, Umehara T, Sasaki $K$ et al. Real-time imaging of histone H4K12specific acetylation determines the modes of action of histone deacetylase and bromodomain inhibitors. Chem Biol 2011; 18: 495-507

57 Rao P, Benito E, Fischer A. MicroRNAs as biomarkers for CNS disease. Front Mol Neurosci 2013; 6: 39

58 Fischer A, Sananbenesi F, Mungenast A et al. Targeting the correct HDAC (s) to treat cognitive disorders. Trends Pharmacol Sci 2010; 31: 605617
59 Zhang ZY, Schluesener HJ. Oral administration of histone deacetylase inhibitor MS-275 ameliorates neuroinflammation and cerebral amyloidosis and improves behavior in a mouse model. J Neuropathol Exp Neurol 2013; 72: 178 - 185

60 Cao $P$, Liang $Y$, Gao $X$ et al. Administration of MS-275 improves cognitive performance and reduces cell death following traumatic brain injury in rats. CNS Neurosci Ther 2013; 19: 337-345

61 Agis-Balboa RC, Pavelka Z, Kerimoglu C et al. Loss of HDAC5 impairs memory function: implications for Alzheimer's disease. J Alzheimers Dis 2013; $33: 35-44$

62 Göttlicher M, Minucci S, Zhu Pet al. Valproic acid defines a novel class of HDAC inhibitors inducing differentiation of transformed cells. EMBO J 2001; 20: 6969-6978

63 Kilgore M, Miller CA, Fass DM et al. Inhibitors of class 1 histone deacetylases reverse contextual memory deficits in a mouse model of Alzheimer's disease. Neuropsychopharmacology 2010; 35: 870-880

64 Tariot PN, Schneider LS, Cummings J et al. Alzheimer's Disease Cooperative Study Group. Chronic divalproex sodium to attenuate agitation and clinical progression of Alzheimer disease. Arch Gen Psychiatry 2011; 68: 853-861

65 Krützfeldt J, Rajewsky N, Braich $R$ et al. Silencing of microRNAs in vivo with 'antagomirs'. Nature 2005; 438: 685-689

66 Kluiver J, Slezak-Prochazka I, Smigielska-Czepiel K et al. Generation of miRNA sponge constructs. Nature Methods 2012; 58: 113-117

67 Whitehead KA, Langer $R$, Anderson DG. Knocking down barriers: advances in siRNA delivery. Nat Rev Drug Discov 2009; 8: 129-138

68 Alvarez-Erviti L, Seow Y, Yin H et al. Delivery of siRNA to the mouse brain by systemic injection of targeted exosomes. Nat Biotechnol 2011; 29: $341-345$ 